\title{
A Rare Case of Broad Ligament Fibroid: A Case Report
}

\author{
Kamala Verma ${ }^{1}$, Santosh Kumari ${ }^{2}$
}

\begin{abstract} and radiological difficulties in differentiating against an ovarian tumor. on the left side. MRI pelvis showed similar findings. IVP was normal. soft tissue tumor-leiomyoma. role in confirming its diagnosis.

Clinical significance: We present this case because of its rarity and the diagnostic difficulties it posed.

Keywords: Broad ligament, Laparotomy, Leiomyoma.

Journal of South Asian Federation of Menopause Societies (2019): 10.5005/jp-journals-10032-1168
\end{abstract}

Background: The broad ligament is the commonest extrauterine site for occurrence of leiomyoma. Because of its rarity, it poses both clinical

Case description: We are presenting a rare case of leiomyoma of broad ligament in a 45-year-old female with complaints of lower abdominal lump, pain, and menstrual abnormality. On clinical examination, there was a 18-week-old firm, nontender, and mobile mass extending to the umbilicus. Ultrasound pelvis showed a large hypoechoic lesion of $17 \mathrm{~cm} \times 11 \mathrm{~cm} \times 10.3 \mathrm{~cm}$ with internal vascularity in pelvis predominantly

Result: Laparotomy showed a huge false left-sided broad ligament leiomyoma measuring $17 \times 15 \mathrm{~cm}$. Histopathology confirmed it to be a

Conclusion: Broad ligament leiomyomas mimic ovarian tumors on clinical and radiological examination and may pose difficulties in differentiating both. Its diagnosis is difficult on clinical and radiological features owing to its unusual presentation. Thus histopathology plays an important

\section{INTRODUCTION}

\section{Background}

Leiomyoma is the most common benign neoplasm of the uterus and the female genital tract accounting for approximately $20-30 \%$ of cases in females of reproductive age group. Leiomyoma can be intrauterine or extrauterine. Among the extra uterine fibroids, broad ligament fibroids are the most common to occur although its overall incidence is extremely rare at $<1 \%{ }^{1}$

Other extrauterine sites are the round ligament, ovarian ligament and the ovaries. Broad ligament fibroids can be primary (true) or secondary (false). ${ }^{2}$ True broad ligament fibroid arises from tissues in the broad ligament itself, uterine vessels and ureter lying medial to the tumor. False broad ligament fibroid arises from the uterus but grows laterally between the two layers of the broad ligament and retains its attachment to the uterus. Uterine vessels and ureter lie lateral to the false broad ligament fibroid. It often coexists with the fibroids of the uterus. ${ }^{3}$ Clinically broad ligament fibroids may manifest as extrauterine pelvic masses that compress the urethra, bladder neck, ureter or bowel producing symptoms of varying degrees of urinary and bowel dysfunction. It may also lead to chronic pelvic pain and menstrual irregularities when it coexists with intrauterine myoma as in our case.

Here we are reporting a rare case of huge broad ligament leiomyoma with diagnostic difficulties in differentiating it from an ovarian tumor. Ovarian malignancy should be ruled out prior to a planned surgery.

\section{Case Description}

We report the case of a 45-year-old lady admitted from the gynecological OPD with complaints of mass per abdomen for two years and abdominal discomfort for the past 3 months. It was gradual in onset and gradually progressed to the present enormous size of up to umbilicus in the past 3 months. She
${ }^{1}$ Department of Obstetrics and Gynaecology, Sardar Patel Medical College, Bikaner, Rajasthan, India

${ }^{2}$ Department of Obstetrics and Gynaecology, Sardar Patel Medical College and Associated Group of Hospitals, Bikaner, Rajasthan, India

Corresponding Author: Santosh Kumari, Department of Obstetrics and Gynaecology, Sardar Patel Medical College and Associated Group of Hospitals, Bikaner, Rajasthan, India, Phone: +91 8005781329, e-mail: bhalgodara@gmail.com

How to cite this article: Verma K, Kumari S. A Rare Case of Broad Ligament Fibroid: A Case Report. J South Asian Feder Menopause Soc 2019;4(1):37-41.

Source of support: Nil

Conflict of interest: None

developed a dull aching abdominal pain associated with heaviness in the lower part of her abdomen. There were no disturbances in her bladder and bowel function. There was a history of prolonged heavy menstrual flow for the past one year. There was no history of weight loss or jaundice. She had two normal vaginal deliveries. Last child birth was 18 years back. There were no history of tubectomy and no family history of fibroid, ovarian, breast or colonic tumor. On physical examination patient was afebrile and hemodynamically stable. According to the abdominal examination, there was a uniform mass of 16-18 weeks old gravid uterus which appears to be arising from the pelvis. There were no dilated/engorged veins over the mass and visible peristalsis. On palpation tumor was firm in consistency, nontender, and mobile in the horizontal direction and with regular margins. Lower pole could not be reached. According to the speculum examination cervix and vagina appeared healthy and pushed toward right. No abnormal discharge. According to the vaginal examination, cervix was firm, deviated to right. Uterus was bulky, mobile, firm, and nontender and the mass moves with

(c) The Author(s). 2019Open Access This article is distributed under the terms of the Creative Commons Attribution 4.0 International License (https://creativecommons. org/licenses/by-nc/4.0/), which permits unrestricted use, distribution, and non-commercial reproduction in any medium, provided you give appropriate credit to the original author(s) and the source, provide a link to the Creative Commons license, and indicate if changes were made. The Creative Commons Public Domain Dedication waiver (http://creativecommons.org/publicdomain/zero/1.0/) applies to the data made available in this article, unless otherwise stated. 
cervical movement. Left and posterior forniceal fullness present with no tenderness.

\section{Investigations}

Her ultrasound abdomen and pelvis showed a large hypoechoic lesion of $17 \mathrm{~cm} \times 11 \mathrm{~cm} \times 10.3 \mathrm{~cm}$ with internal vascularity in pelvis predominantly on the left side. The left ovary is not separately visualized. Uterus and right ovary are normal in size, shape, and echotexture. No evidence of ascites and lymphadenopathy.

MRI pelvis showed a well-defined lesion in left adnexa of $16.7 \times 16.1 \times 9 \mathrm{~cm}$ which appeared hyperintense on T2W while hypointense on T1W image (Figs 1 to 3 ). The lesion displaced uterus to the right side. Small cystic areas are seen within the lesion. Perilesional flow voids are seen. Uterus and right ovary are normal in size and ehotexture. Few well-defined hypointense lesions seen on anterior wall and fundus of uterus largest measuring $17 \times 15 \mathrm{~mm}$ were likely to be intramural fibroids.

Her routine blood investigations and serum biochemistry were within normal limits. CA 125 was also normal. She was worked up for laparotomy. IVP was done which showed no obstruction of ureter or no dilatation of renal calyces.

\section{Intraoperative Findings}

The intraoperative diagnosis was huge broad ligament myoma and multiple intrauterine myoma. Intraoperatively, following a midline longitudinal incision with extension over the umbilicus, a $17 \times 15 \mathrm{~cm}$ sized fibroid was noted in the left broad ligament. Uterus was pushed to the right side. Uterus was normal, left fallopian tube and round ligament looked grossly normal, and both ovaries were normal. Two smaller intramural fibroids were noted on the anterior aspect of the uterus. Careful dissection was done to prevent ureteric injuries. Excision of tumor with total abdominal hysterectomy and bilateral salpingo-oopheretomy was done (Fig. 4). It was a false broad ligament fibroid. Cut section of tumor showed a gray white mass with multiple cystic areas filled with a mucoid and gelatinous material.

\section{Histopathology}

On gross examination, a single large globular soft tissue mass measuring $17 \times 15 \mathrm{~cm}$ was found. Cut section showed mucoid and cystic areas. Microscopic examination showed leiomyoma with cystic degeneration.

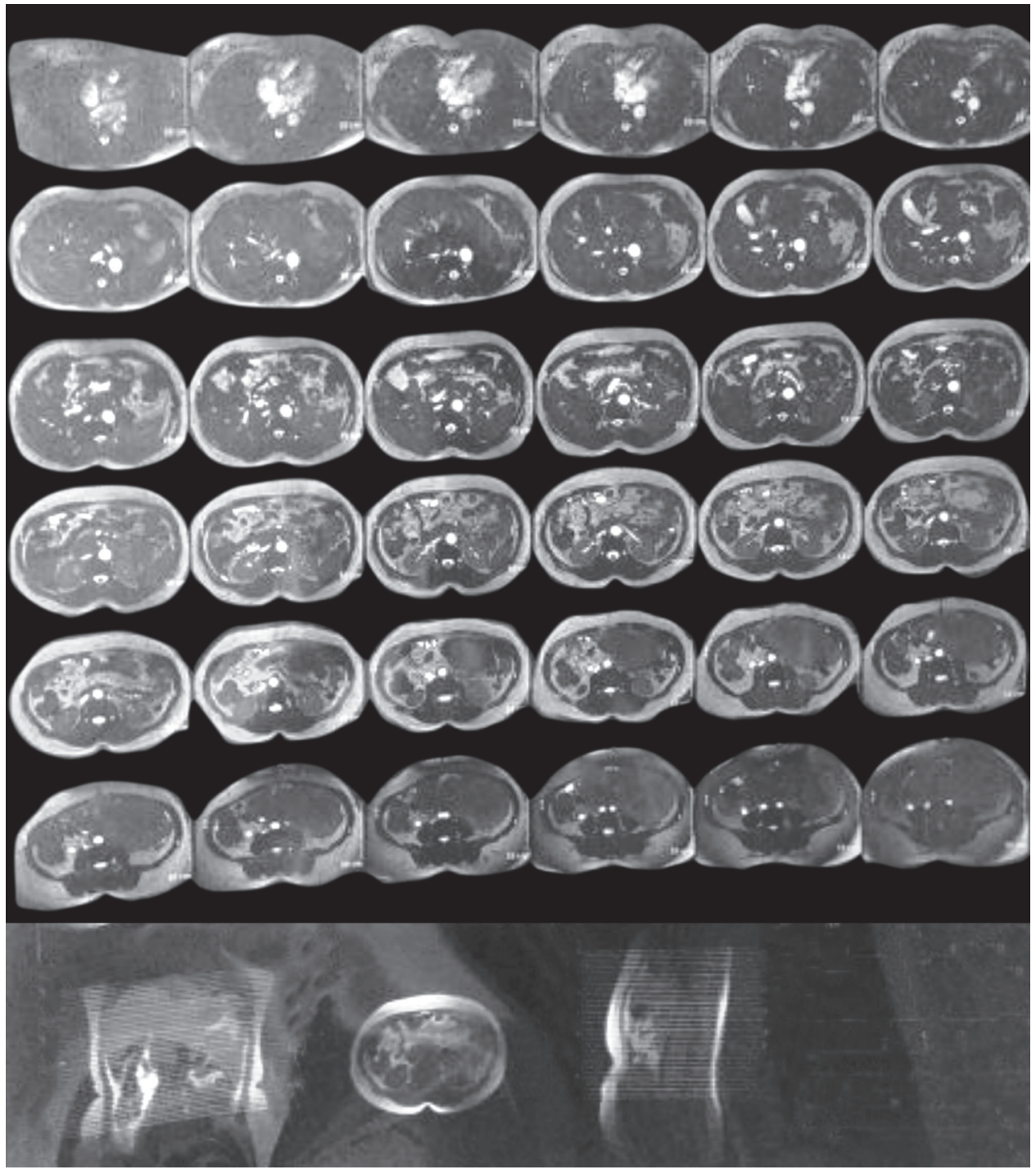

Fig. 1: MRI pelvis T1W image showing hypointense lesion S/O broad ligament fibroid 


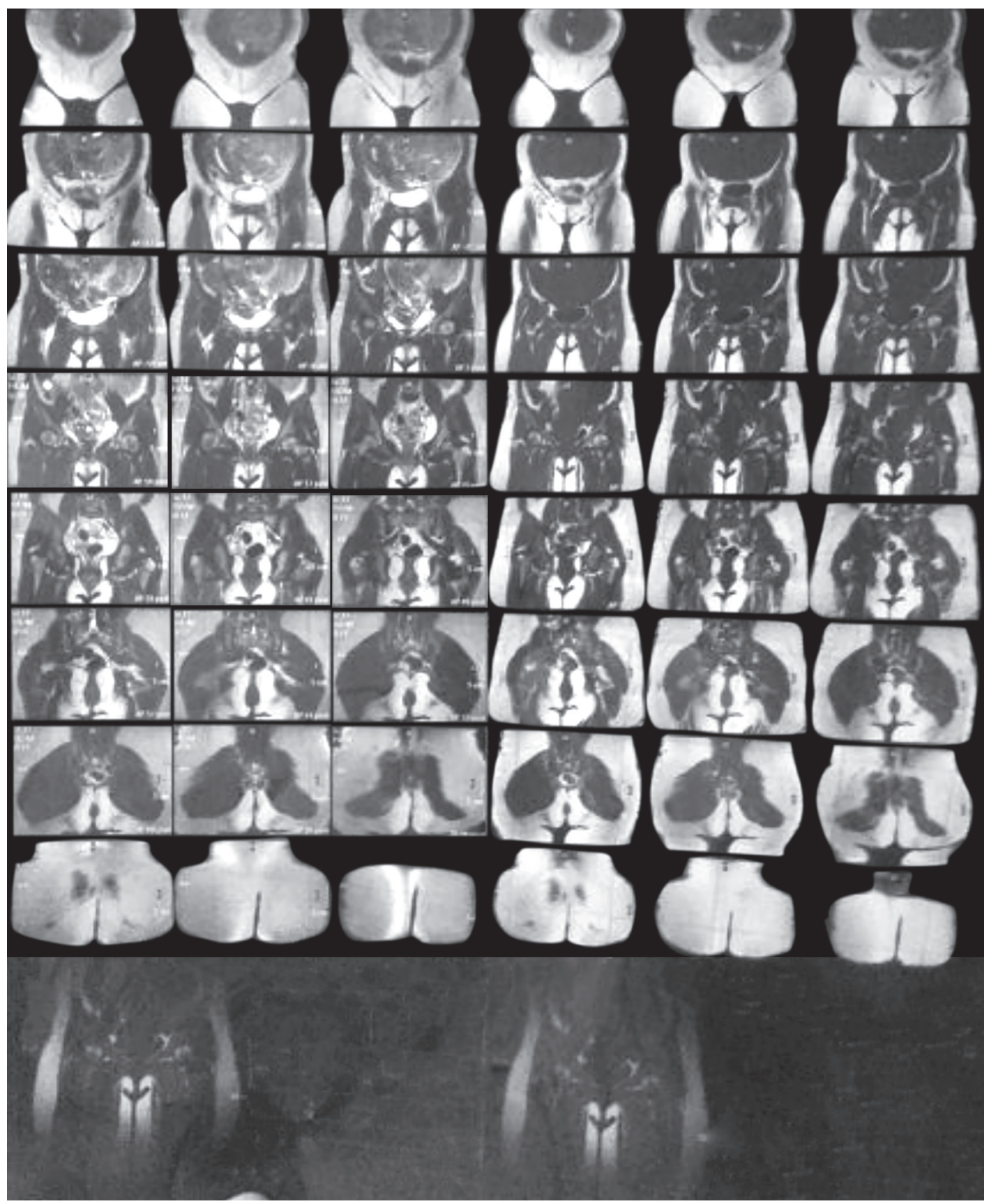

Fig. 2: MRI pelvis T2W image coronal view showing broad ligament fibroid

Histopathology of uterus with cervix, bilateral fallopian tubes and ovaries were unremarkable.

Postoperative period was uneventful and the patient was discharged on postoperative day 8 .

\section{Discussion}

Broad ligament is a two layered fold of peritoneum connecting the sides of uterus to lateral walls of pelvis and its floor. Among the broad ligament tumors, epithelial tumors are the most common type whereas mesenchymal tumors are rare. Among the mesenchymal tumors, the most common one is leiomyoma. ${ }^{4}$ It has been suggested that leiomyomas which are adherent to broad ligament, originate from hormonally sensitive smooth muscle elements of broad ligament itself. Broad ligament leiomyoma can originate from the uterus and invade the broad ligament (false) or it can originate from broad ligament itself (true). These benign tumors are usually asymptomatic. However, if the leiomyoma reaches significant size, it can potentially compress the surrounding pelvic structures and manifest clinically with various signs and symptoms. The location of tumors often determines the various symptoms. ${ }^{5}$ If allowed to reach an enormous size, it can present with pressure symptoms, pelvic pain, bladder and bowel dysfunction. Intrauterine fibroid on the other hand in addition to pressure symptoms often presents with menstrual abnormalities and dysmenorrhea. The case presented here had both chronic lower abdominal pain and heaviness in the lower abdomen likely to have been caused by the huge broad ligament fibroid and menstrual abnormalities likely to be due to the 


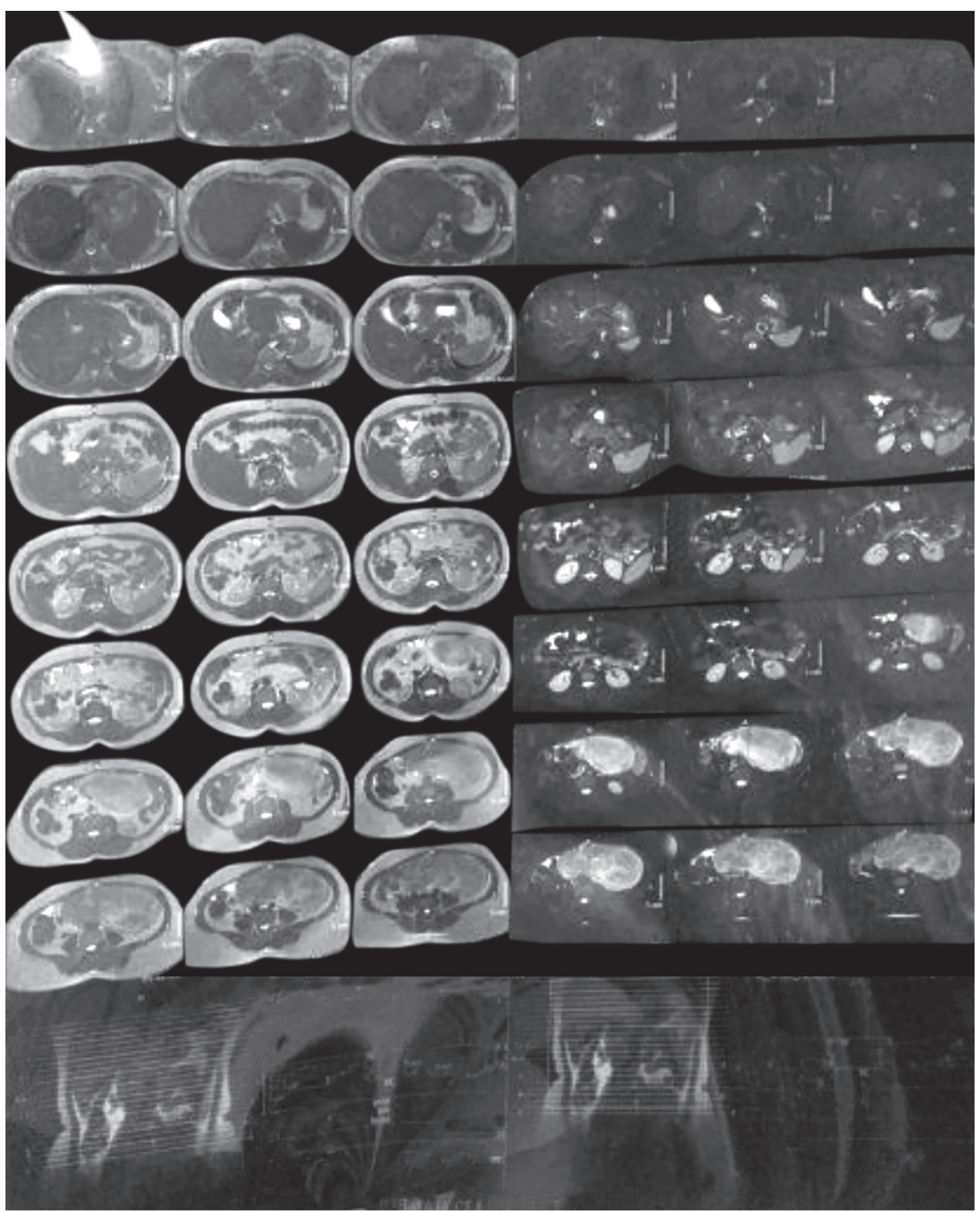

Fig. 3: MRI pelvis T2W image transverse view showing broad ligament view

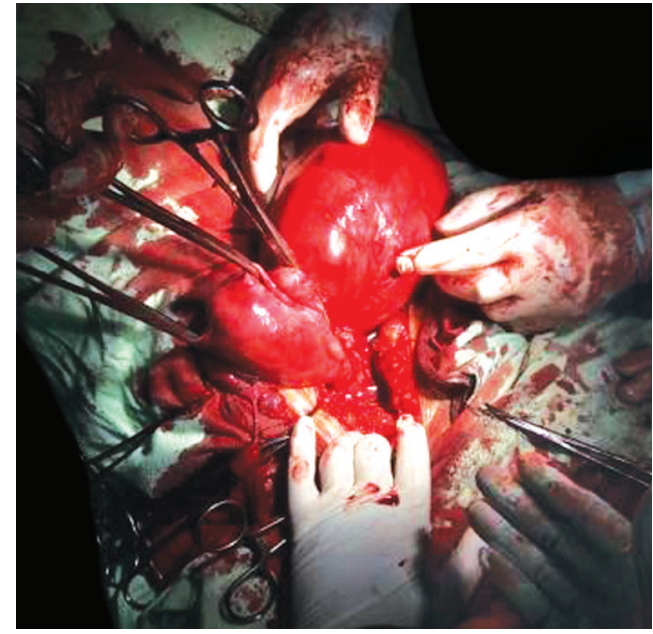

Fig. 4: Intraoperative image of the left huge broad ligament fibroid. Left ovary and fallopian tube are grossly normal intrauterine fibroid. It was important for any adnexal masses to discriminate between benign and malignant nature of the lesion in the preoperative period for optimal patient management. The differential diagnosis for broad ligament leiomyoma includes ovarian masses (benign or malignant), tubo-ovarian masses, broad ligament cyst and lymphadenopathy. In our case on clinical examination and ultrasonography it was suspicious of an ovarian tumor or subserosal fibroid. The serum level of cancer antigen CA-125 was done which was in normal range. Leiomyomas may be single or multiple. In our case, there was a single mass in broad ligament with small intrauterine leiomyomas. Broad ligament leiomyomas have the potential to grow to large size. ${ }^{6}$ When they grow to a large size, secondary changes may occur which includes degeneration, infection, hemorrhage, and necrosis. The cystic changes in lesion mimic the metastatic malignant ovarian tumors. ${ }^{7}$ Primary leiomyosarcoma in broad ligament is rarely reported. ${ }^{8}$ Degenerative changes in the leiomyomas are considered to be 
due to inadequate blood supply. There is no particular relationship between symptoms and the incidence of degenerative changes.

\section{Conclusion}

Broad ligament leiomyomas mimic ovarian tumors on clinical and radiological examination and may pose difficulties in differentiating both. It should be kept as an important differential diagnosis of solid adnexal or ovarian masses. The diagnosis of broad ligament leiomyoma is difficult on clinical and radiological features owing to its rarity and unusual presentation. Thus histopathology plays an important role in confirming its diagnosis. It is also not uncommon for it to coexist with uterine myoma.

\section{References}

1. Kumar P, Malhotra N. Jeffcoate's Principles of Gynaecology, 7th ed., New Delhi: Jaypee Brothers; 2008. p. 492.
2. Jeffcoate N. Tumors of corpus uteri. In: Batla N. ed. Jeffcoate's Principles of Gynaecology, 6th ed., Delhi: Arnold Publication; 2001. pp. 466-497.

3. Neha G, Manisha L. A Rare Case of Giant Broad Ligament Fibroid with Cervical Fibroid Mimicking Ovarian Tumour. Int J Recent Trends Sci Technol 2014;10:208-209.

4. Thor AD, Young RH, et al. Pathology of fallopian tube, broad ligament, peritoneum and pelvic soft tissues. Hum Pathol 1991;22:856-867. DOI: 10.1016/0046-8177(91)90174-N.

5. Stewart EA. Uterine Fibroids. Lancet 2001;357:293-298. DOI: 10.1016/ S0140-6736(00)03622-9.

6. Godbole RR, Laksmi KS, et al. Rare case of giant broad ligament fibroid with myxoid degeneration. J Sci Soc 2012;39:144-146.

7. Rajanna DK, Pandey V, et al. Broad ligament fibroid mimicking as ovarian tumor on ultrasonography and computed tomography scan. J Clin Imaging Sci 2013;3:8. DOI: 10.4103/2156-7514.107912.

8. Duhan N, Singh S, et al. Primary leiomyosarcoma of the broad ligament: case report and review of literature. Arch Gynecol Obstet 2009;279:705-708. DOI: 10.1007/s00404-008-0777-2. 